\title{
The comparison of insulin resistance frequency in patients with recurrent early pregnancy loss to normal individuals
}

\author{
Kotanaie Maryam ${ }^{1}$, Zinatossadat Bouzari ${ }^{1,2}$, Zahra Basirat $^{3,4^{*}}$, Mehrdad Kashifard ${ }^{5}$ and Mahtab Zeinal Zadeh ${ }^{3,4}$
}

\begin{abstract}
Background: Patients with $\geq 3$ recurrent spontaneous miscarriages are classified as having RSM. Polycystic ovary syndrome (PCOS) is associated with insulin resistance (IR). The purpose of this study is to evaluate the association of IR and RMS.

Methods: Present case- control prospective study was performed on 100 women in control group (with a history of at a live birth and no history of one more abortion) and study group (with a history of $\geq 3$ RMS) who were not diabetes and PCOS. Two groups matched in base of age and body mass index. Blood was withdrawn from the case and control patients for the determination of the fasting blood glucose (FG), fasting insulin (FI) levels and ultrasonography was performed on all the patients.
\end{abstract}

Results: The observed differences between age, FG and FG to Fl ratio levels in case and control groups were not significant $(p>0.05$ ) but it was significant about fasting insulin $(p=0.0119)$. Fl of $<20 \mu \mathrm{u} / \mathrm{m} /$ or $\geq 20 \mu \mathrm{u} / \mathrm{m} /$ in case and control group was significant (Chi-square: 4.083, p: 0.0433, odds ratio: 4.4386, Cl95\% $=1.1541$ to 17.0701 ), whereas the difference between absolute and proportional frequency of patients with FG to FI ratio of $<4.5$ and $\geq$ 4.5 in case and control groups was not significant (Chi-square: $2.374, p=0.123$ ).

Conclusion: Current study showed that in women with RPL, in Iranian race like Americans, frequency of insulin resistance in high, therefore there is a probability of the degree of insulin resistance in women with RPL.

\section{Background}

Recurrent pregnancy loss (RPL) is estimated to occur in $2 \%-4 \%$ of reproductive -age couples [1]. Patients with $\geq$ 3 recurrent spontaneous miscarriages are classified as having RSM. An RSM remains is a very disturbing event to the affected patients by this health problem; they are always anxious to find the underlying reasons for their miscarriages. This is also a major challenge to the treating physicians [2]. It is a major hazard in pregnancy, both for naturally conceived and those after assisted reproductive technology (ART) treatment [3].

Intensive researches including immunological and genetic studies are still in progress to illustrate the cause of RSM [2]. Chromosome anomaly, uterine malformations or anomalies, hypothyroidism, cervical in

\footnotetext{
* Correspondence: Zahra_basirat@yahoo.com

${ }^{3}$ Infertility and Reproductive Health research center, Babol University of Medical Science, Babol, Iran

Full list of author information is available at the end of the article
}

competence, anti phospholipid syndrome, bacterial infections and polycystic ovary syndrome (PCOS) are some of the etiological factors associated with RSM [4], [5]. There are some reports of high RSM rates in over weight/obese infertile women treated by ART [6].

Other reports are the condition of PCOS which is probably linked with obesity [7]; this may be due to the high prevalence of overweight/obesity in PCOS women [8]. PCOS is associated with insulin resistance (IR)independent of total or fat- free body mass which can be a key factor behind the link between PCOS/obesity and the risk of spontaneous abortion [3,9]. IR is often increased in 40\% women with PCOS [4],[10], and hyperinsulinemia is an etiological factor in the pathogenesis of PCOS [11]. Further studies detected a correlation between increasing insulin resistance and fasting insulin level, with PRL [12]. The exact mechanism of how IR leads to RSM is unknown [2]. IR can be diagnosed by the determination of the fasting glucose to fasting 
insulin ration, a ratio of $<4.5$ being diagnostic of IR [13]. In the present study we were tried to evaluate the association of IR and recurrent pregnancy loss in our area.

\section{Methods}

The present case- control prospective study evaluated between March 2007 and March 2008 in the Department of Obstetrics and Gynecology, Shahid Yahyanejad Hospital, in Iran. At the first 114, patients were evaluated, however, 7 women from the study group and 7 women from control group were eliminated due to not recon tact us.

Fifty non pregnant women with $\geq 2$ RSM with history, positive serum $B$-HCG and ultrasound documentation of pregnancy, with same sex partner without history of diabetes, were classified as case group. Pregnancy loss was defined as any natural abortion occurring before 24 weeks gestation [1]. Fifty women in reproductive age, non-diabetic, had at least one live birth and/or maximum of one were selected as the control group. The patients of two groups matched in terms of age and body mass index. For the case groups, they completed an evaluation for RSM that included: hysterosalpingogram, serum prolactin, TSH, midluteal serum P, lupus anticoagulant, IgG, IgM, IgA anticardiolipin, antiphosphatidyl serine antibodies, karyotypes on both partners and cervical cultures for Chlamydia, urea plasma, mycoplasma.

Patients with the history of non - consecutive miscarriages, ectopic pregnancy, molar pregnancy, diabetes, multiple partner, PCOS, and current pregnancy were out of the study. The objective and design of this study were explained to all the patients and whole data was completely secret. A written informed consent was obtained from all the participants. The Ethics Committee of the Babol Medical Science University approved the study protocol.

The diagnosis of PCOS patients were based upon the history of having chronic oligoamenorrhea (oligoamenorrhea and amenorrhea were defined as $\leq 6$ menses per year and no menses for 1 year retrospectively), clinical and biochemical hyperanderogenism (hirsutism, and severe acne and high levels of total or free testosterone, and rostenedione and DHEAS), abnormal hormonal tests of FSH, LH and those found on ultrasound to contain 12 or more follicles measuring 2 to $9 \mathrm{~mm}$ in diameter.

Blood was withdrawn from the case and control patients for the determination of the fasting blood glucose (FG) and fasting insulin (FI) levels. The patients had 12-hour overnight fast before the blood was extracted from the patients between 8:00 and 10:a.m. on the day of the test [14]. Fasting serum insulin was determined by a ECL method (Kit: Gbas, Hitachi 2010, Japan). Glucose concentration was determined by the glucose oxides method(Kit: Pars Azmon, Hitachi 2010, Japan). The insulin resistance is diagnosed by the determination of the fasting glucose to fasting insulin ratio, a ratio of $<4.5$ or insulin level of $\geq 20 \mu$ unit being diagnostic of insulin resistance[12].

\section{Statistical analysis}

The complete data from the two groups were subjected to statistical analysis using Chi-square for determination of a variable between two groups and t-test for the comparison between two qualities using SPSS package.

\section{Result}

At first, 114 patients were evaluated but 7 women from the case group and 7 women from the control group were eliminated due to not recon tact us. Mean and standard deviation of case group were 28.15 and \pm 4.76 , respectively (between 19 and 41 years).

Based from the results taken from independent Sample t-test about comparison of the mean of age, fasting blood glucose (FG), fasting insulin (FI) and FG to FI ratio levels in case and control is shown in- Table 1-, the observed differences between age, FG and FG to FI ratio levels in case and control groups were not significant $(p>0.05)$ but it was significant about fasting insulin $(p=0.0119)$.

Based from the results taken from Chi-square about the comparison of absolute and comparative frequency of patients with FI of $<20 \mu u / m l$ or $\geq 20 \mu u / m l$ in case and control group was significant (Chi-square:4.083, $p$ : 0.0433 , odds ratio: 4.4386 , CI95\% $=1.1541$ to 17.0701 ), whereas the difference between absolute and proportional frequency of patients with FG to FI ratio of $<4.5$ and $\geq 4.5$ in case and control groups was not significant

\begin{tabular}{|c|c|c|c|c|c|c|}
\hline Parameter & Groups & Frequency & Mean & $\begin{array}{l}\mathrm{SD} \pm \\
\text { Mean }\end{array}$ & $T$ & $\begin{array}{c}P \\
\text { value }\end{array}$ \\
\hline \multirow[t]{2}{*}{ Age } & Control & 50 & $28 / 28$ & $4 / 77$ & $\begin{array}{c}0 / \\
568\end{array}$ & $0 / 5715$ \\
\hline & Case & 50 & $28 / 02$ & $4 / 57$ & & \\
\hline \multirow[t]{2}{*}{ FG } & Control & 50 & $90 / 56$ & $10 / 12$ & $\begin{array}{l}1 / \\
810\end{array}$ & 0/0735 \\
\hline & Case & 50 & $94 / 12$ & $9 / 34$ & & \\
\hline \multirow[t]{2}{*}{$\mathrm{Fl}$} & Control & 50 & $12 / 23$ & $5 / 64$ & $\begin{array}{c}2 / \\
565\end{array}$ & 0/0119 \\
\hline & Case & 50 & $15 / 20$ & $5 / 82$ & & \\
\hline \multirow[t]{2}{*}{$\begin{array}{c}\mathrm{FG} \text { to } \mathrm{FI} \\
\text { ratio }\end{array}$} & Control & 50 & $8 / 40$ & $3 / 26$ & $\begin{array}{c}1 / \\
726\end{array}$ & 0/0876 \\
\hline & Case & 50 & $7 / 26$ & $3 / 28$ & & \\
\hline
\end{tabular}


(Chi-square: 2.374, $p=0.123$ ). Based from the result taken Chi-square test, the difference between absolute and proportional frequency of patients with IR in case and control groups was significant with $(p: 0.0248$, (Table 2).

The comparison of absolute and proportional frequency of patients with $\mathrm{BMI} \geq 27$ and patients with BMI $<27$ and the difference between absolute and proportional frequency of patients with IR in both groups with $P=0.75$ was not significant.

\section{Discussion}

Recurrent pregnancy loss (RPL) is estimated to occur in $2-4 \%$ of reproductive - age couples [1]. A multitude of etiologic factors have been proposed, including: $3.1 \%$ genetic (balanced translocations) 20.2\% endocrinologic (elevated levels of serum TSH. hyper prolactinemia, luteal phase defects), $21.6 \%$ anatomic (congenital uterine anomalies, Asherman's syndrome, leionyomata, uterinePolyps or septa), 25\% immunologic (elevated levels of anticardiolipin or antiphospholipid antibodies, lupus anticoagulant) and 5.8\%. microbidogic factors [12,15].

Further studies detected a correlation between increasing insulin resistance and fasting insulin level, with PRL. These findings caused the investigation of IR in women with other causes of PRL.Craig et al in their study showed increased prevalence of insulin resistance in women with a history of at least 2 pregnancy losses [12].

Present study was performed on 100 women incase and control group (with a history of at least a live birth and no history of one more abortion) and the study group (with a history of $\geq 2 \mathrm{RPL}$ ) who were not diabetic and PCOS. Mean and standard deviation of age were $28.18 \pm 4.67$ (at least 19 and maximum 41 years old). A group of 21-25 years old with 28 patients (28\%) was the most frequent group. And in the age group of 26-30 years old with 26 patients (26\%) also depending upon our study, the average of the age height, weight and BMI between case group and control group did not have significant difference which was not unpredictable

Table 2 Comparison of absolute and comparative frequency of patients with insulin resistance (IR) in case and control groups

\begin{tabular}{llccc}
\hline Groups & & IR & Non-IR & T \\
\hline Control & Absolute frequency & 4 & 46 & 100 \\
\cline { 2 - 5 } & Proportional frequency & $\% 8$ & $\% 92$ & $\% 100$ \\
\hline Case & Absolute frequency & 12 & 38 & 100 \\
\cline { 2 - 5 } & Proportional frequency & $\% 24$ & $\% 76$ & $\% 100$ \\
\hline Total & Absolute frequency & 16 & 84 & 100 \\
\cline { 2 - 5 } & Proportional frequency & $\% 16$ & $\% 84$ & $\% 100$
\end{tabular}

Chi-square $=5.038 P=0.0248$ Odds ratio $=3.6486$

$\mathrm{Cl} 95 \%=1.0855$ to 12.2642 based on matching control and case. According to Craig et al results [12], also the frequency of patients with high level of FI (FI $\geq 20 \mu u / m l)$ in case group was significantly more than the control group (22.45\% versus $6.21 \%$, odds Ratio 4.4386, CI: $95 \%=1.1541$ to 17.0701 ) and frequency of patients with IR is significantly higher than the frequency of these patients in control group (22.49\% versus $8.16 \%$, odds Ratio 3.6486 , CI $95 \%=$ 1.0855 to 12.2642 ), but no significant difference was detected between the patients with proportion of FG to FI ratio(G:I) less than 4.5 between case and control group. These findings were in harmony with Craig et al. findings. In Crag et al., study's FI in case group was significantly more than control group and the proportion of patients with high FI and IR in case group was significantly more than control group [12].

In our study and Craig et al., study, also versus other studies the patients with undefined spontaneous miscarriages were investigated, all the patients with RPL were under survey [16,17]. May be because of this the most patients has more than one reason for miscarriage $[12,15-18]$. Consequently there is more probability for presenting more than one cause of miscarriage in the patient in which finding of one responsible cause stops finding of other cause. Therefore in raft of studied patients IR was not detected. Craig et al; study's showed in 11 patients of 38 patients with RPL (28.9\%) there is one more cause except insulin resistance for RPL [12].

Different concepts of insulin resistance may cause the different results of this study and other studies. In current study we used Craig et al procedure for insulin resistance concept which means fasting insulin of $\geq 20$ $\mu u / m l$ and/or of fasting blood sugar to fasting insulin ratio less than 45. Legro etal., concluded fasting G: I (glucose/Insulin) ratio is maybe useful as a screening test for insulin resistance in obese non-Hispanic white PCOS women, with the highest sensitivity and specificity. The exact mechanism of insulin resistance for RPL is not completely clear but there is some explanation about it [19].

It is possible that PCOS women have a hostile uterine environmental milieu which causes decrease conception and/or early miscarriages. Elevated PAI-I (plasminogen activator inhibitor-I, an endogenous inhibitor of fibrinolysis) levels have been independently associated with recurrent miscarriages in PCOS women [20].

Metformin enhances luteal phase uterine vascularity and blood flow and reduce the rate of first trimester spontaneous abortions [21,22]. Metformin therapy throughout pregnancy in women with PCOS reduces the otherwise high rate of first-trimester spontaneous abortion [14]. Some in vitro data demonstrate that insulin and glucose modulate glucose transporter at the cellular level of the placentaltrophoblast. Thus maternal 
insulin and glycemic status may influence the expression of GLUTI [23], also some researchers showed the rule of PA inhibitor which is elevated in women with RPL $[24,25]$. Increases by elevation of insulin level and decreases with insulin, sensitizator drugs [14,26]. Maybe elevation of this component changes the trombotic process and causes failure in placenta and at last loss of pregnancy [12].

\section{Conclusion}

Current study showed that in women with RPL, in Iranian race like Americans, frequency of insulin resistance in high, therefore there is a probability of the degree of insulin resistance in women with RPL. We recommend that glucose and fasting insulin levels in serum should be measured in all women with RPL. Also another, study in other similar studies in different population is necessary to confirm these findings and the effect of reluctant plasma glucose concentrations drugs in women with history of RPL which should be evaluated in further studies.

Present study involved in some limitation for example, this study done just in one hospitals in Babol city and doesn't cover all hospitals. We suggest to future researchers to cover big population.

\section{Source of finding}

Shahid Yahyanejad Hospital

\section{Acknowledgements}

Authors would like to thank the Research's Vice chancellor of the University for their Financial Support. We also appreciate the collaborations of the Health's Vice chancellor for as well as all the staff working in the related health centers.

\section{Author details}

'Obstruction \&Gynecology Department, Babol University of Medical Science, Babol, Iran. ${ }^{2}$ Member of Cellular \& Molecular Biology Research Center, Babol University of Medical Science, Babol, Iran. ${ }^{3}$ Infertility and Reproductive Health research center, Babol University of Medical Science, Babol, Iran.

${ }^{4}$ Department of Internal Medicine, Ayat Allah Rouhani Hospital, Babol University of Medical Sciences, Babol, Iran. ${ }^{5}$ Anestesio Department, Babol University of Medical Science, Babol, Iran.

\section{Authors' contributions}

Each author has participated actively and sufficiently in this study. MK conceived the idea and design of the study, interpretation of data, and drafted the manuscript. ZB conceived the idea and design of the study. ZB and ZB made substantial contribution to analysis and interpretation of data. AN and MM have made contribution to collecting of data and editing. Each author revised critically the manuscript and provided final approval of the version to be published.

\section{Competing interests}

The authors declare that they have no competing interests.

Received: 11 December 2011 Accepted: 9 March 2012

Published: 9 March 2012
References

1. Kutteh WH: Recurrent pregnancy loss. In Textbook of reproductive medicine.. 2 edition. Edited by: Carr BR, Blackwell RE. Stamford, CT: Appleton and Lange; 1998:679-692.

2. Diejomaoh M, Jirous J, Al-Azemi M, Gupta M, Al-Jaber M, Farhat R, Mohd A: Insulin resistance in women with recurrent spontaneous miscarriage of unknown etiology. Med Princ Pract 2007, 16(2):114-118.

3. Li Tian, HuanShen, Qun Lu, Norman JRobert, Jim Wang: Insulin resistance increases the risk of spontaneous abortion after assisted reproduction technology treatment. J Clin Endocrinol Metab 2007, 92(4):1430-1433.

4. Diejomaoh M, Jirous J, Al-Azemi M, Baig S, Gupta M, Tallat A: The relationship of recurrent spontaneous miscarriage to reproductive failure. Med Princ Pract 2003, 12:107-111.

5. Dunaif A, Segal KR, Futterweit W, Dobrajansky A: Profound peripheral insulin resistance, independent of obesity in polycystic ovary syndrome. Diabetes 1989, 38:1165-1174.

6. Wang JX, Davies MJ, Norman RJ: Obesity increases the risk of spontaneous abortion during infertility treatment. Obes Res 2002, 10:551-554.

7. Hamilton-Fairley D, Kiddy D, Watson H, Paterson C, Franks S: Association of moderate obesity with a poor pregnancy outcome in women with polycystic ovary syndrome treated with low dose gonadotrophin. $\mathrm{Br} J$ Obstet Gynaecol 1992, 99:128-131.

8. Wang JX, Davies MJ, Norman RJ: Polycystic ovarian syndrome and the risk of spontaneous abortion following assisted reproductive technology treatment. Hum Reprod 2001, 16:2006-2009.

9. Dhindsa $G$, Bhatia $R$, Dhindsa M, Bhatia $V$ : Insulin resistance, insulin sensitization and inflammation in polycystic ovarian syndrome. $J$ Postgrad Med 2004, 50(2):140-144.

10. Anttila L, Karjala K, Penttila RA, Ruutiainen L, Ekblad U: Polycystic ovaries in women with gestational diabetes. Obstet Gynecol 1998, 92:13-6.

11. Ehrmann DA, Liljenquist DR, Kasza K, Azziz R, Legro RS, Ghazzi MN: Prevalence and predictors of the metabolic syndrome in women with polycystic ovary syndrome. J Clin Endocrinol Metab 2006, 91:48-53.

12. Legro RS, Finegood D, Dunaif A: A fasting glucose to insulin ratio is a useful measure of insulin sensitivity in women with polycystic ovary syndrome. L Clin Endocrinol Metab 1998, 83(8):2694-2698.

13. Glueck CJ, Phillips H, Cameron D, Sieve-Smith L, Wang P: Continuing metformin throughout pregnancy in women with polycystic ovary syndrome appears to safely reduce first-trimester spontaneous abortion: a pilot study. Fertil Steril 2001, 75(1):46-52.

14. Craig LB, Ke RW, Kutteh WH: Increased prevalence of insulin resistance in women with a history of recurrent pregnancy loss. Fertil Steril 2002, 78(3):487-490.

15. Carmina E, Lobo RA: Use of fasting blood to assess the prevalence of insulin resistance in women with polycystic ovary syndrome. Fertil Steril 2004, 82(3):661-665.

16. Kutteh WH, Carney JL: Etiologic factors in women with a history of recurrent pregnancy loss. ObstetGynecol 1999, 93:42S.

17. Stephenson MD: Frequency of factors associated with habitual abortion in 197 couples. Fertil Steril 1996, 66:241-291.

18. Stephenson MD: Frequency of factors associated with habitual abortion in 197 couples. Fertil Steril 1996, 66(1):24-9.

19. Legro RS, Finegood D, Dunaif A: A fasting glucose to insulin ratio is a useful measure of insulin sensitivity in women with polycystic ovary syndrome. J Clin Endocrinol Metab 1998, 83(8):2694-8.

20. Glueck CJ, Wang P, Fontaine RN, Sieve-Smith L, Tracy T, Moore SK: Plasminogen activator inhibitor activity: an independent risk factor for the high miscarriage rate during pregnancy in women with polycystic ovary syndrome. Metabolism 1999, 48:1589-1595.

21. Glueck CJ, Wang P, Goldenberg N, Sieve-Smith L: Pregnancy outcome among women with polycystic ovary syndrome treated with metformin. Hum Reprod 2002, 17:2858-2864.

22. Jakubowicz DJ, luorno MJ, Jakubowicz S, Roberts KA, Nestler JE: Effects of metformin on early pregnancy loss in the polycystic ovary syndrome. $J$ Clin Endocrinol Metab 2002, 87:524-529.

23. Gordon MC, Zimmerman PD, Landon MB, Gabbe SG, Kniss DA: Insulin and glucose modulate glucose transporter messenger ribonucleic acid expression and glucose uptake in trophoblasts isolated from firsttrimester chorionic villi. Am J Obstet Gyneocol 1995, 173(4):1089-1097. 
24. Gris JC, Neveu S, Mares P, Biron C, Hredon B, Schved JF: Plasma fibrinolytic activators and their inhibitors in women suffering from early recurrent abortion of unknown etiology. J Lab Clin Med 1993, 122(5):606-615.

25. Gris JC, Ripar-Neveu S, Maugard C, Tailland ML, Brun S, Courtieu C, Biron C, et al: Respective evaluation of the prevalence of haemostasis abnormalities in unexplained early recurrent miscarriages. The Nimes Obstetricians and Heamatologists (NOHA) study. Thromb Haemost 1997, 77(6):1069-1103

26. Glueck CJ, Papanna R, Wang P, Goldenberg N, Sieve-Smith L: Incidence and treatment of metabolic syndrome in newly referred women with confirmed polycystic ovarian syndrome. Metabolism 2003, 52(79):908-915.

doi:10.1186/1756-0500-5-133

Cite this article as: Maryam et al.: The comparison of insulin resistance

frequency in patients with recurrent early pregnancy loss to normal individuals. BMC Research Notes 2012 5:133.

\section{Submit your next manuscript to BioMed Central} and take full advantage of:

- Convenient online submission

- Thorough peer review

- No space constraints or color figure charges

- Immediate publication on acceptance

- Inclusion in PubMed, CAS, Scopus and Google Scholar

- Research which is freely available for redistribution

Submit your manuscript at www.biomedcentral.com/submit 